\title{
FREQUENCY ESTIMATION DERIVED FROM A DYNAMICAL SYSTEM ANALYSIS
}

\author{
Cyril Hory, Michael Ting and Alfred Hero \\ Department of Electrical Engineering and Computer Science, \\ University of Michigan, \\ 1301 Beal Avenue Ann Arbor, MI. 48109 USA.
}

\begin{abstract}
The main concern of this paper is to build a method of characterization and identification of a dynamical system with several degrees of freedom when only one observation record is available. The analysis of the number of degrees of freedom provided by performing a Principal Component Analysis leads to an eigendecomposition-based method. The link between the proposed method and the MUSIC algorithm is then drawn. This approach gives a new insight on the use of this type of methods.
\end{abstract}

\section{INTRODUCTION}

Performing single spin detection with Magnetic Resonance Force Microscopy (MRFM) raises questions ranging from theoretical to experimental physics. Apart from the understanding of how quantum and classical mechanics systems interact [5], an important issue is the characterization of a nonlinear dynamical system from a single observation. That is the problem this communication proposes to tackle. A MRFM device consists of coupling a single electron in resonance in a magnetic field with a ferromagnetic cantilever. Under hypothesis $H_{0}$, no spin is present. The cantilever is a harmonic oscillator whose natural frequency depends on the magnetic field gradient. The motion of the cantilever is described by a dynamical system with two degrees of freedom. The introduction of the nonlinear coupling term of the cantilever with a single spin under hypothesis $H_{1}$ induces an increasing of the number of degrees of freedom from two to five. A detection scheme is proposed in [10] that performes a parallel tracking of the output signal under both hypothesis. This method requires a simulation of the systems of dynamical equations, and thus the estimation of all the degrees of freedom. Here the spin detection task is reduced to determining how many degrees of freedom are necessary to

\footnotetext{
Research partially supported by DARPA MOSAIC program under ARO contract DAAD19-02-C-0055.
}

describe the system from the only observation of the output of the cantilever.

We first recall that the Takens Embedding method provides a phase space reconstructed trajectory of a dynamical system from a single observation [9]. Under suitable approximations, it can be shown [1], that the coupling of the single spin with the cantilever results in a shift of the natural frequency. Thus, the characterization task turns out to be the detection and estimation of this shift. Working with a 5-dimensional phase space, we propose to determine the actual number of degrees of freedom by performing a Principal Component Analysis. This approach gives a new insight on the eigendecomposition-based methods of frequency estimation [4], [6]. We finally present a comparison of the proposed algorithm with the well-known MUSIC algorithm [3], [7].

\section{ANALYSIS OF A DYNAMICAL SYSTEM}

Consider a system of $p$ differential equations with variables $d_{1}(t), d_{2}(t), \ldots, d_{p}(t)$

$$
\left\{\begin{aligned}
\frac{d_{1}(t)}{d t} & =\Phi_{1}\left(d_{1}(t), d_{2}(t), \ldots, d_{p}(t)\right), \\
\frac{d_{2}(t)}{d t} & =\Phi_{2}\left(d_{1}(t), d_{2}(t), \ldots, d_{p}(t)\right), \\
& \vdots \\
\frac{d_{p}(t)}{d t} & =\Phi_{p}\left(d_{1}(t), d_{2}(t), \ldots, d_{p}(t)\right) .
\end{aligned}\right.
$$

Suppose the only observation available is the component $d_{1}(t)$. There exists a mapping from the actual phase space

$$
\left(d_{1}(t), d_{2}(t), \ldots, d_{p}(t)\right),
$$

to the space spanning the set of $p$ delayed versions of the observation [9]

$$
\left(d_{1}(t), d_{1}(t-\tau), \ldots, d_{1}(t-(p-1) \tau)\right) .
$$

This space is called the reconstructed phase space. Parameter $\tau$ is called the embedding delay. Fraser shows in [2] that an optimal choice of $\tau$ in terms 
of information redundancy is the first zero of the auto-correlation function of $d_{1}(t)$. That is how we define $\tau$ here. The actual number $p$ of degrees of freedom of the dynamical system is the dimension of the trajectory. If unknown, it can be estimated from the Principal Component Analysis (PCA) of the reconstructed phase space. The estimation of $p$ is then given by the number of non-null eigenvalues of the covariance matrix of the reconstructed phase space.

Consider that the available observation is the discrete signal

$$
x[n]=d_{1}\left(n T_{s}\right)+\nu[n], \quad n=0 \ldots N-1
$$

where $T_{s}$ is the sampling period and $\nu[n]$ is the measurement white Gaussian noise with variance $\sigma^{2}$. Note

$$
x_{n}^{l}=[x[n], x[n+l], \ldots, x[n+(p-1) l]]^{T},
$$

the $l$-times under-sampled segment of the observation. Performing PCA of the phase space consists of deriving the eigendecomposition of the $p \times p$ covariance matrix

$$
C^{l}=\frac{1}{P} \sum_{n=0}^{P-1} x_{n}^{l} x_{n}^{l T},
$$

where $P=N-l(p-1)$ is the number of undersampled segments. The under-sampling rate $l$ is related to the embedding delay $\tau$ by

$$
l T_{s}=\tau+\varepsilon T_{s},
$$

where $\varepsilon \in(-1 / 2,1 / 2)$ is the error to the estimation of the embedding delay, due to the discretization of the observation. As $P$ tends to infinity, $C^{l}$ tends to the matrix $\Gamma^{l}=E\left\{x_{n}^{l} x_{n}^{l T}\right\}$. Assuming wide sense stationary dynamics, this matrix is of Toeplitz form with the element in the $k$ th diagonal $\Gamma_{i, k}^{l}=\Gamma_{1+(i-1) l, 1+(k-1) l}^{1}$.

\section{THE PHASE-SPACE TRAJECTORY OF THE MRFM DEVICE}

The dynamics of a cantilever in a MRFM device is described by a system of $p=5$ equations which variables are the position and speed of the cantilever tip and the three components of the spin [1], [10]. The available observation $d_{1}$ is the position of the cantilever measured by a laser interferometer. Under hypothesis $H_{0}$ (no spin), the cantilever is an harmonic oscillator and the number of degrees of freedom reduces to $p=2$. The variable $d_{1}$ is, in this case, a sinusoid with frequency $f_{0}$ equal to the natural frequency of the oscillator:

$$
H_{0}: \quad d_{1}[n]=A \sin \left(2 \pi f_{0} n T_{s}+\psi\right) .
$$

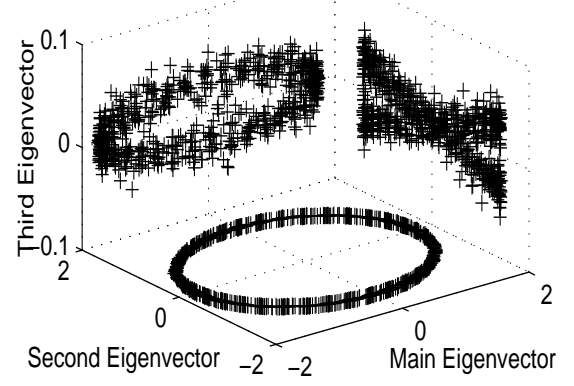

Fig. 1. Projection of the phase space trajectory on the three first eigenvector space. The central frequency is $f_{0}=7000 \mathrm{~Hz}$ and the shift is $\delta f_{0}=30$ $\mathrm{Hz}$.

In MRFM, the amplitude $A$ of the signal and phase $\psi$ are parameters controlled by the user. We assume they are known with values $A=1$ and $\psi=$ 0 . Thus the frequency is the only parameter to be estimated. The trajectory in the 5-dimensional reconstructed phase space is an ellipsoid with radii and orientation depending on $f_{0}$. This trajectory lies on a two-dimensional subspace.

The cantilever is driven by an external force which produces a cyclic adiabatic inversion $(\mathrm{CAI})$ of the spin moment. It is shown in [1] that the effect of the CAI on the spin-cantilever coupling can be approximated as cyclically alternating positive and negative shift $\delta f_{0}$ of the natural frequency of the cantilever:

$$
f=f_{0} \pm \delta f_{0} .
$$

Thus, under $H_{1}$, the trajectory in the phase space is composed of two ellipsoids with different radii and different orientations (see figure 1). Under $H_{1}$, the trajectory spans more than two dimensions.

\section{THE EIGENDECOMPOSITION-BASED METHOD}

The detection scheme we develop consists of tracking the orientation of the trajectory over a moving time window. This orientation is provided by the eigenvectors of the covariance matrix $C^{l}$ rather than by the eigenvalues. As the output of the cantilever is a sinusoid, its period is approximately $4 l$ where $l$ is the discrete embedding delay defined by expression (5). We propose to perform a PCA of the reconstructed trajectory on successive sequences $\left[x_{n}^{l}, x_{n+1}^{l}, \ldots, x_{n+M-1}^{l}\right]$. The eigenvectors of $C^{l}$ are asymptotically unbiased estimators of the eigenvectors $\gamma_{1}^{l}, \gamma_{2}^{l}, \ldots, \gamma_{p}^{l}$ of $\Gamma^{l}$ [3]. The eigenvector $\gamma_{1}^{l}$ corresponding to the highest eigen- 
value is expressible as:

$$
\gamma_{1}^{l}=\left[e\left(f_{0}\right)^{4}, e\left(f_{0}\right)^{3}, e\left(f_{0}\right)^{2}, e\left(f_{0}\right), 1\right]^{T},
$$

where $e\left(f_{0}\right)=\exp \left\{-i 2 \pi f_{0} l T_{s}\right\}$. The PCA of the phase space provides an unbiased estimation of $\hat{e}\left(f_{0}\right)$. The estimated frequency $\hat{f}_{0}$ is the angular position of $\hat{e}\left(f_{0}\right)$ :

$$
\hat{f}_{0}=\frac{1}{2 \pi l T_{s}} \Im\left\{\log \left\{\hat{e}\left(f_{0}\right)\right\}\right\},
$$

where $\Im\{x\}$ is the imaginary part of the complex value $x$.

\section{COMPARISON WITH MUSIC}

The frequency estimation method described in the previous section is based on the eigendecomposition of the covariance matrix $\Gamma^{l}$. It can be seen as a member of the class of subspace methods [6]. One of the most popular method belonging to this class is the well-known MUSIC algorithm introduced first by Pisarenko in [4] for frequency estimation and extended to the problem of direction of arrival estimation (see for example [3] or [7] for analysis of performances of MUSIC and related algorithms)

Unlike our proposed method, MUSIC is based on the eigendecomposition of the entire covariance matrix $\Gamma^{1}$ of the data following model (2) with

$$
d_{1}[n]=\sum_{j=1}^{k} A_{j} \exp \left\{i 2 \pi f_{j} n T_{s}+\psi_{j}\right\}
$$

sum of $k$ cisoids. The estimates $\hat{f}_{j}$ of the frequencies are solution of

$$
a^{*}(f) G^{1} G^{1 *} a(f)=0,
$$

where

$$
a(f)=\left[1, \ldots, \exp \left\{i 2 \pi f(m-1) T_{s}\right\}\right]^{T}
$$

and $G^{1}$ is the matrix of the noise subspace eigenvectors:

$$
G^{1}=\left[\gamma_{k+1}^{1}, \ldots, \gamma_{m}^{1}\right]
$$

namely, the eigenvectors with eigenvalues $\lambda_{k+1}=$ $\ldots=\lambda_{m}=\sigma^{2}$.

For a comparison with the MUSIC algorithm, a sinusoid of $f_{0}=7000 \mathrm{~Hz}$ embedded in an additive white Gaussian noise of variance $\sigma^{2}=1$ has been simulated. Figure 2 presents the projection of the trajectory of the signal on the span of the three first eigenvectors $\left(\gamma_{1}^{l}, \gamma_{2}^{l}, \gamma_{3}^{l}\right)$ of the under-sampled covariance matrix. Subsequently, the signal is projected on the signal subspace estimated by the MUSIC algorithm. The phase space trajectory of this filtered signal is projected on the space $\left(\gamma_{1}^{l}, \gamma_{2}^{l}, \gamma_{3}^{l}\right)$. One can observe that the projections of the two signals on the span of the two first eigenvectors space are similar. However, the trajectory of the filtered signal is nearly orthogonal to $e_{3}$. The same observation can be made for the two last eigenvectors. This shows that the signal-space derived from the eigendecomposition of the under-sampled covariance matrix is bi-dimensional, as predicted by the analysis of the dynamical system.
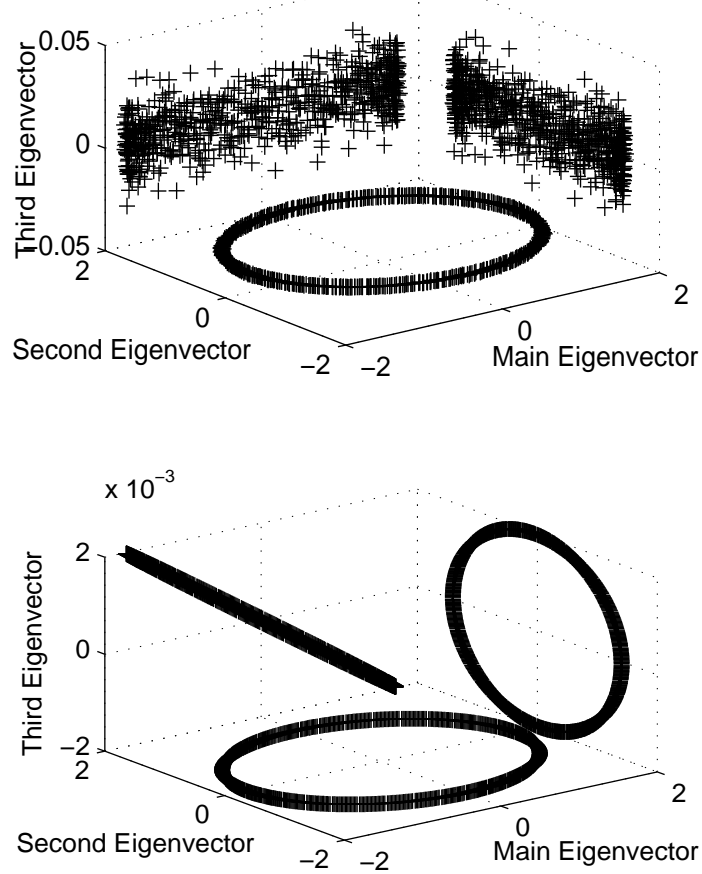

Fig. 2. Projections of the phase space trajectories on the three first eigenvector space of a sinusoid (top) and of its projection on the MUSIC signal space (bottom). The frequency is $f_{0}=7000 \mathrm{~Hz}$.

A comparative study of the performances of MUSIC and our proposed method is presented on figure 3. The frequency of a cisoid embedded in a white Gaussian noise of variance $\sigma^{2}=1$ is estimated by both methods using $C^{l}$ for several under sampling rates $l$. The proposed method is applied with a constant length of snapshot $m$ and decreasing dimension $p$ as $l$ increases such that $m=$ $(p-1) l+1$.

The bias of the MUSIC frequency estimator is almost constant over the delay $l$. However, the variance of MUSIC increases as $l$ increases. This is due to the decreasing of the dimension $p$, given that 

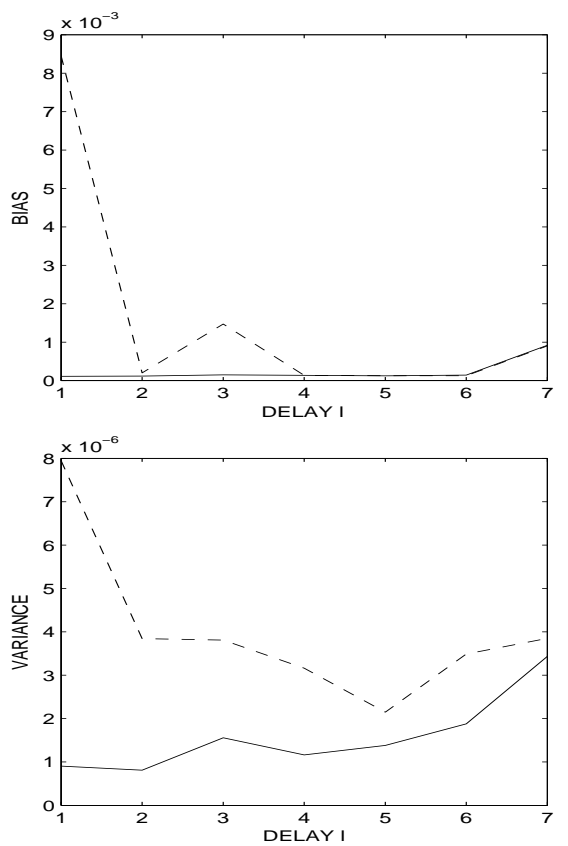

Fig. 3. Comparison between frequency estimates from MUSIC (plain lines) and from the proposed estimator (dashed lines) in terms of the delay $l$. The signal is a cisoid of normalized frequency $f_{0}=$ 0.05 with length $N=200$ points. Each snapshot is of length $m=21$ points before sub-sampling. The signal to noise ratio is $S N R=0 \mathrm{~dB}$.

the variance of MUSIC does not depend on the normalized frequency [8]. Note that the performance of the method derived from the Takens embedding improves when $l$ increases. The bias of Takens embedding is similar to the bias of MUSIC when $l>4$, while the variance is still slightly higher. However one can see that the variance of the proposed method reaches a minimum value when $l=$ 5 . This delay is the value suggested by Fraser in [2].

\section{CONCLUSION}

The analysis of the phase space of a dynamical system has led us to the formulation of an eigendecomposition-based method of frequency estimation. This approach gives a new insight into the use of this type of method. The proposed one is directly derived from dynamical system analysis. The output signal model is non-stationary. Therefore, a straight application of MUSIC or related estimators is not valid. The use of the under-sampled covariance matrix suggested by the Takens embedding approach is better suited to non-stationary signal analysis and more specifically, estimation of shift in frequency.

\section{REFERENCES}

[1] G. P. Berman, D. I. Kamenev, and V. I. Tsifrinovich. Stationary cantilever vibrations in the oscillating cantilever-driven adiabatic reversals - magnetic resonance force microscopy technique. Quantum physics, http://arxiv.org/list/quant-ph/0203, March 2002.

[2] A. M. Fraser. Information and entropy in strange attractors. IEEE trans. on information theory, 35(2):245-262, March 1989.

[3] M. Kaveh and A. J. Barabell. The statistical performance of the MUSIC and the minimum-norm algorithms in resolving plane waves in noise. IEEE trans. on acoustics, speech and signal proc., 34(2):331-341, April 1986.

[4] V. F. Pisarenko. The retrieval of harmonics from a covariance function. Geophys. J. R. astr. Soc., 33:347-366, 1973.

[5] J. Sidles. Path integrals over measurement amplitudes: Practical quantum foundations for signal processing and control. arXiv:quant-ph, http://arxiv.org/list/quantph/0211?100, November 2002.

[6] P. Stoica and R. Moses. Introduction to Spectral Analysis. Prentice Hall, 1997.

[7] P. Stoica and A. Nehorai. MUSIC, maximum likelihood and cramer-rao bound. IEEE trans. on acoustics, speech and signal proc., 37(5):720-741, May 1989.

[8] P. Stoica and T. Söderström. Statistical analysis of MUSIC and subspace rotation estimates of sinusoidal frequencies. IEEE trans. on signal proc., 39(8):1836-1847, August 1991.

[9] F. Takens. Detecting strange attractors in turbulence. In Proceedings of the Symposion on Dynamical Systems and Turbulence, University of Warwick, 1979-1980, volume 1, pages 366-381. Springer, Berlin., 1981.

[10] M. Ting and A. Hero. Piece-wise kalman filtering for estimation of a MRFM cantilever signal. In Proceedings of SSP03, Saint-Louis, Mo., USA, October 2003. 